\title{
Einführung
}

Die ,postpolitische Stadt'

Boris Michel

Nikolai Roskamm

In der kritischen Stadtforschung hat sich in den letzten Jahren der begriffliche Rahmen der ,neoliberalen Stadt' für die Analyse und Beschreibung aktueller urbaner Entwicklungen durchgesetzt (Künkel/Mayer 2012, Leitner/Peck/Sheppard 2007, Brenner/Theodore 2002). Dabei wird Neoliberalismus nicht allein in einem engen ökonomischen Sinne verstanden, sondern viele Autor_innen greifen auch auf gouvernementalitätstheoretische Perspektiven (Foucault 2004a; 2004b) zum besseren Verständnis stadtpolitischer Regime zurück (vgl. Füller/Michel 2012). Damit werden in einem in einem relativ weiten, durch (post-)marxistische und (post-)strukturalistische Theorien beeinflussten Forschungsfeld etwa Fragen der Privatisierung und Kommodifizierung städtischer Räume mit Fragen der Kontrolle und (Selbst-)Steuerung ,neoliberalisierter 'Subjekte zusammengebracht. Dieses Verständnis ermöglicht es, auf den ersten Blick sehr unterschiedliche Prozesse in einem gemeinsamen begrifflichen Rahmen zu fassen.

Zunehmende Aufmerksamkeit in der kritischen Stadtforschung findet dabei die Debatte, in der die Frage nach dem eigentlichen Gehalt des Politischen gestellt und auf die Differenz zwischen ,der Politik' und ,dem Politischen' fokussiert wird (Lefort 2007 [1999], Lacou-Labarthe/Nancy 1997 [1990], Laclau 1990). Mit dem Begriff des Postpolitischen, der aus eben jenem Umfeld der politischen Theorie stammt, wird die derzeitige ,reale Politik' als eine Politik kritisiert, die zum einen das konflikthafte Wesen des Politischen verdrängt habe und zum anderen darauf abziele, das offene, störende, nicht festgelegte und verändernde Moment abzuschaffen, das dem Politischen ebenfalls zu eigen ist.

Mit dem Themenheft „Die postpolitische Stadt?“ möchten wir uns an beiden Debatten - an der Debatte zur neoliberalen Stadt und an der Debatte zum Begriff des Politischen/Postpolitischen - beteiligen und kritische Stadtforschung verstärkt mit den sogenannten neuen Theorien des Politischen [1] in einen Dialog bringen. Ein solche Zusammenkunft erscheint uns aus verschiedenen Gründen vielversprechend zu sein: Unsere Ausgangsthese lautet, dass genau der Bereich der politischen Theorie, auf den wir mit dem Begriff der ,postpolitischen Stadt' verweisen, zu einer 
Aktualisierung kritischer Stadtforschung beitragen und damit der Blick auf Widerständigkeiten, Verfahren des Regierens und neue Formen staatlicher Politik geschärft werden kann. Unser Ziel ist es dabei nicht, die fruchtbaren Debatten um die neoliberale Ordnung des Städtischen für beendet oder für unzulässig zu erklären. Vielmehr geht es uns darum, eine Ergänzung vorzuschlagen. In diesem Heft wollen wir ausloten, inwiefern und an welchen Stellen durch aktuelle politische Theorie inspirierte und informierte Forschungsansätze in der Lage sind, die traditionellen und etablierten Ansätze der kritischen Stadtforschung zu bereichern. Aber wir möchten ebenso beleuchten, wo möglicherweise Widersprüche und Unvereinbarkeiten zwischen beiden Denkrichtungen bestehen.

Mit unserem Themenheft schließen wir an eine Diskussion an, die im englischsprachigen Raum bereits seit einigen Jahren in den urban studies geführt wird (vgl. Purcell 2013, Dikeç 2013; 2005, Allmendinger/ Haughton 2012). Nicht zuletzt ist der Import der These vom Postpolitischen in die kritische Stadtforschung dem Geographen Erik Swyngedouw zu verdanken, dessen Text „The post-political city“ (2007) wir für diese Ausgabe ins Deutsche übersetzt haben. Swyngedouw geht von der Annahme aus, dass die Herausbildung eines „urban post-political arrangement“ parallel zum Aufstieg einer Neoliberalisierung des Städtischen verläuft. Zentrales Moment dieses Regierens sei ein Verschwinden von Dissens und Konflikt zugunsten einer Reihe von Technologien des Regierens, „that fuse around consensus, agreement, accountancy metrics and technocratic environmental management" (Swyngedouw 2009: 604). Die neoliberale Stadt, so Swyngedouws Erkenntnis, ist die postpolitische Stadt, das Konzept der Postpolitik materialisiert sich in ihr. Diese These liefert den roten Faden für unser Themenheft.

\section{Das Konzept des Postpolitischen}

Um in unser Themenheft einzuführen, möchten wir an dieser Stelle zunächst die theoretische Ausgangslage der Debatte zum Postpolitischen und deren ideengeschichtliche Herkunft skizzieren. Eine solche Debatte ist in der kritischen Stadtforschung unseres Erachtens nicht nur zu begrüßen, sondern auch gar nicht zu vermeiden, denn kritische Stadtforschung - so lautet unsere Kurzformel - braucht kritische (Stadt-)Theorie. Unsere Frage nach der ,postpolitischen Stadt' setzt genau hier an.

Eingeführt wurde der Begriff der Postpolitik Ende der 1990er Jahre von Slavoj Žižek (2010 [1999]) - dem umstrittenen slowenischen Popstar der politischen Philosophie - mit explizitem Bezug auf den französischen Theoretiker Jacques Rancière. Žižek folgt Rancières Konzeption des Politischen als eigene für sich stehende Sphäre und erweitert dessen Typologie des Politischen. Während bei Rancière (2002 [1995] 73f.) ,ArchePolitik' auf den Ursprung von polis und politike verweist, ,Para-Politik durch eine Polizei- und Wettstreitlogik bestimmt wird und ,Meta-Politik ${ }^{`}$ das Modell eines wissenschaftlich-technologischen, instrumentellen Verfahrens repräsentiert, basiert Žižeks Begriff von Postpolitik auf dem Modell der geschäftlichen Verhandlung und des „strategischen Kompromisses“ (Žižek 2010: 272f.). Postpolitik ist für Žižek die Bezeichnung des Typus 
des Politischen, in dem die „Kollaboration voraufgeklärter Technokraten (Ökonomen, Meinungsforscher) mit liberalen Multikulturalisten“ den „Konflikt globaler ideologischer Entwürfe“ ersetzt. Über den Prozess des Aushandelns von Interessen, so formuliert es Žižek, wird ein mehr oder weniger allgemeiner Konsens erreicht. Postpolitik plädiere folglich immer „für die Notwendigkeit, die alten ideologischen Unterscheidungen hinter sich zu lassen" und sich - ausgestattet mit dem notwendigen Expert_innenwissen und dem freien Meinungsaustausch, der die jeweils konkreten Bedürfnisse und Forderungen der Menschen zur Sprache bringt - den neuen Problemen zu stellen (ebd.: 273).

Als postpolitisch bezeichnet Žižek also den dominanten politischen Modus des gegenwärtigen Kapitalismus und dessen liberal-demokratische Ideologie. Als Inbegriff und einflussreiche Verfechter_innen solcher postpolitischen bzw. postdemokratischen Rationalitäten gelten in der Debatte insbesondere New Labour und die deutsche Sozialdemokratie unter Gerhard Schröder. Mit ihrer Selbstdefinition als Modernisierer der politischen Linken, so Colin Crouch (2008), hätten New Labour und die Protagonist_innen des ,Dritten Weges' ein Konzept von Politik ohne Gegner durchgesetzt. Anschließend an Žižek, der Silvio Berlusconi als herausragenden Vertreter eines postpolitischen Projekts anführt, lässt sich mithin feststellen, dass in den meisten europäischen Staaten politische Auseinandersetzungen zwischen den ehemaligen Volksparteien als Auseinandersetzungen darüber aufzufassen sind, welche es vermag, sich selbst als die wirklich postideologische Partei und ihre Kontrahenten als Vertreter einer vergangenen ideologischen Epoche darzustellen (Žižek 2002: 303).

Aufgegriffen wird das Konzept der Postpolitik von Chantal Mouffe, die sich ebenfalls deutlich gegen die „postpolitische Vision“ (2007: 64) einer kosmopolitischen, liberal-demokratischen und konsensorientierten Perspektive positioniert. Mouffe, die zusammen mit Ernesto Laclau bereits in den 1980er Jahren eine einschlägige Hegemonie- und Antagonismustheorie vorgelegt hat (Laclau/Mouffe 1991), geht in ihren späteren Arbeiten zum Begriff des Politischen (Mouffe 1993; 2000) einen anderen Weg: Im Rückgriff auf den antiken, nietzscheanisch aktualisierten agon (Wettstreit) als Alternative zu (oder als Ausweg vor) einem radikalen Antagonismus (Kampf, Krieg) schlägt sie ein Konzept vor, mit dem sie die radikale Negativität des Antagonismus (vgl. Marchart 2013) entschärfen möchte. Mouffe erklärt die Zähmung des Antagonismus in einen agon zur eigentlichen Aufgabe der Demokratie.[2]

Mouffe, die zwischenzeitlich auch in der deutschsprachigen Stadtforschung recht breit rezipiert wurde, bezieht sich explizit auf Carl Schmitt und dessen Schrift Der Begriff des Politischen (1932). Und tatsächlich findet sich bei Schmitt, dem ersten Staatsjuristen des NS-Regimes, eine ganz ähnliche zeitkritische Diagnose: Schmitt beklagte in den 1930er Jahren die allgemeine ,Entpolitisierung' durch die ständige Ausweitung der Bereiche des Liberal-Demokratischen und des Ökonomischen (vgl. Schmitt 1933). Allgemein beruht Schmitts Konzept des Politischen auf seiner berühmten Bestimmung der politischen Sphäre als Schauplatz der Auseinandersetzung zwischen Freund und Feind. Das ,Wesen des 
Politischen' wird nach Schmitt genau durch diese Unterscheidung bestimmt: als Bereich antagonistischer Beziehungen. Einerseits, das sollte bei der Einordnung von Schmitts Ausführungen nicht vergessen werden, wurde seine Theorie damit höchst anschlussfähig für die Feind-Konstruktionen der Nationalsozialisten. Andererseits bestimmt Schmitt - wie es Jacques Derrida (2002: 336) formuliert hat - bis heute mit seiner „hellsichtigen“ Verbindung zwischen dem Politischen und dem Antagonistischen die Grundzüge weiter Teile der politischen Theorie, und zwar gerade derjenigen Teile, die sich unter dem begrifflichen Konzept des Postpolitischen versammeln.

Aus einem weiteren Grund ist Schmitts Begriff des Politischen für die zeitgenössische politische Theorie prägend: Schmitt vertritt dezidiert die Ansicht, dass das Politische nicht irgendeine, sondern die entscheidende und privilegierte ontologische Sphäre ist. Und Schmitt formuliert auch, was dem Politischen (und seinen Bestandteilen Freund/Feind) gegenübersteht: das Ökonomische (mit den Bestandteilen profitabel/nichtprofitabel) und das Soziale (mit den Bestandteilen materiell gleich/ungleich). Insgesamt konzipiert Schmitt das Politische als eigenen, von der ,realen Politik' (wo das Soziale und das Ökonomische anzutreffen sind) zu trennenden Bereich und damit ebenso eine Differenz zwischen ,dem Politischen' und ,der Politik'. Postpolitik ist in diesem Rahmen als ein Begriff zu verstehen, der eine Spielart von Politik jenseits des Politischen bezeichnet. Und: Postpolitik ist selbst ein Begriff des Politischen beziehungsweise setzt - Schmitt paraphrasierend - einen Begriff des Politischen voraus.

Eine zweite und von der Schmitt'schen Herkunft zu trennende Traditionslinie der ,neuen Theorien des Politischen' (und daher auch des Postpolitik-Begriffs) sind die Schriften von Hannah Arendt (1994 [1961]; 1960). Auch Arendt unterscheidet zwischen dem Politischen auf der einen und dem Sozialen (und dem Ökonomischen) auf der anderen Seite. Sie besteht dabei ebenfalls auf einem Primat des Politischen und beklagt dessen Niedergang - auch von hier aus ist es nicht weit zu einem Konzept des Postpolitischen. Allerdings unterscheidet sich Arendts Ansatz in einem entscheidenden Punkt von Schmitt: Für Arendt ist das Politische nicht die Sphäre des antagonistischen Kampfes. Im Gegenteil ist das Politische für sie - mit Bezug auf die antike Polis - eine Bühne des gemeinsamen und freien Austausches (also ebenfalls eines agon) unter Gleichen.[3] Ein solches Konzept steht nicht nur im Gegensatz zu Schmitts FreundFeind-Theorie, sondern in gewisser Weise auch im Widerspruch zu den Interventionen der Postpolitik-These: Žižek und Mouffe kritisieren ja genau die Konzeption des Politischen im Sinne eines - wie etwa Mouffe es formuliert - Habermasianischen Seminarraums, in dem freie Kommunikation demokratische Konsense erzielt.

Arendts politische Theorie ist aber dennoch und aus einem anderen Grunde den ,neuen Theorien des Politischen" und der postpolitischen These eng verbunden: Arendt wendet sich nämlich vehement gegen jedes objektivistische und technokratische Verständnis des Politischen, gegen jeglichen Determinismus und jegliche Konzeption von historischer Vorbestimmtheit und damit gegen die Postulierung von Alternativlosigkeit - auch deshalb darf sie bei unserem kurzen Abriss der Debatten um den Begriff der Postpolitik 
nicht fehlen. Dieser Fokus von Arendt führt ebenfalls zum Kern der These des Postpolitischen, mit der sich - auf der theoretischen Metaebene - gegen die Fixierung von Bedeutung und gegen das Herausnehmen von unveränderlichen Bereichen aus der Sphäre des Politischen gewendet wird. Während nämlich das Postpolitische gerade davon lebt zu postulieren, dass Geschichte zu Ende ist und grundlegende Alternativen nicht möglich sind, eröffnet das Politische und sein Bewusstsein der „Geschichtlichkeit des Seins“ (Laclau 1990: 4) vollkommen neue Ansatzpunkte und Perspektiven für radikale Politik.

Die besondere Pointe ist nun, dass die an Arendt explizierte These zwar einerseits mit dem Konzept der neoliberalen Stadt hervorragend zu vereinbaren ist: Nicht nur Swyngedouw hat herausgearbeitet, dass die neoliberale Stadt und die postpolitische Stadt weitgehend identisch sind. Im Kern beider Erzählungen steht die Behauptung eines ökonomischen Determinismus, also die These, dass das Ökonomische das Gesellschaftliche bestimmt und dass es daher letztlich auch keine Alternative zur gegenwärtigen Entwicklung geben kann (vgl. Roskamm 2012). Aber an genau diesem Punkt geraten andererseits die Ansätze der neoliberalen und der postpolitischen Stadt möglicherweise in einen Konflikt. Arendt wirft nämlich dem Marxismus ebenfalls eine deterministische Konzeption von Geschichte vor: einen ökonomischen Determinismus, bei dem gerade nicht das Politische, sondern das Ökonomische den Vorrang hat (zumindest in ,letzter Instanz', wie es bei Althusser heißt) und bei dem die Lösung des Problems bereits im Problem selbst enthalten ist (die Überwindung des Kapitalismus ist dem Kapitalismus immanent). Und hier schließt sich der Kreis: Weil die Tradition der kritischen Stadtforschung - Traditionen, die immer auch „machtdurchtränkte Strukturen“ (Marchart 2010: 24) sind - viel mit einer marxistisch geprägten politischen Ökonomie zu tun hat, beinhaltet unser Ansinnen, die postpolitische These in die Stadtforschung zu importieren, durchaus Potenzial für Kontroversen. Auf der einen Seite treffen sich in der Diagnose der postpolitischen Stadt zwei Kritikrichtungen: die der traditionell auf die Analysen der politischen Ökonomie zurückgreifenden - kritischen Stadtforschung und die der poststrukturalistisch informierten neuen Theorien des Politischen. Beide kritisieren den dominanten Neoliberalismus und dessen marktgläubige Narrative. Auf der anderen Seite geraten beide Denkrichtungen jedoch auf der Bühne, die mit dem Begriff des Postpolitischen errichtet wird, potenziell miteinander in Streit. Politische Ökonomie, so postulieren wichtige Protagonisten der ,neuen Theorien des Politischen', war als Wissenschaft nur möglich, weil der kapitalistischen Akkumulation der Status des Essentiellen zugesprochen wurde (Laclau 1990: 57). Mit der „Fiktion der politisch genannten Ökonomie“ sei zwar eine Kritik an der kapitalistischen Akkumulation angekündigt worden, etabliert habe sich jedoch deren „allgemeine Herrschaft“ (Badiou 2010: 22).

Die ,neuen Theorien des Politischen' beinhalten damit in ihrem Kern eine Perspektive, mit der nicht nur der Neoliberalismus, sondern auch der Marxismus als ökonomistisch kritisiert wird. Deshalb streiten die Theoretiker_innen des Politischen - das ist ein Punkt, der die höchst unterschiedlichen Ansätze vielfach vereint - auch nicht nur für eine Priorität des Politischen gegenüber dem Sozialen und dem Ökonomischen, sondern 
ziehen zu Felde gegen jede deterministische Welt- und Geschichtserklärung - sei es die Erklärung des neoliberalen Endes der Geschichte und des alternativlosen Sieges des Kapitalismus, sei es das im historischen Materialismus vorgezeichnete zwangsläufige Ende des Kapitalismus. Der Begriff der Postpolitik steht also nicht nur für das Primat des Konflikts gegenüber dem Konsens (eine These, der in der kritischen Stadtforschung gerne gefolgt wird), sondern transportiert gleichzeitig ein Kontingenzkonzept, das jeglichen ,letzten Grund' und jedes unumstößliche Fundament (und sei es das eigene) zum Angriffspunkt hat. Geschichte ist nicht vorbestimmt, so lautet das Credo von Arendt und den postfundamentalistischen Denker_innen des Politischen. Dadurch ist in der These der postpolitischen Stadt nicht nur eine Kritik an der neoliberalen Stadt, sondern eben auch der Keim einer Kritik am traditionellen (politökonomischen) Fundament der kritischen Stadtforschung enthalten.

Diese Konstellation - das Zusammentreffen von Konflikt als die neuen Theorien des Politischen mit kritischer Stadtforschung tendenziell verbindendes und Kontingenz als beide Bereiche tendenziell trennendes Element - ist der Kontext unseres Themenheftes. Die Fragen, die in den nachfolgenden Beiträgen verhandelt werden, lauten daher (zumindest latent) immer auch: Worin liegt das „Rätsel des Politischen“(Gauchet/Lefort 1990: 92)? Was ist das Politische? Das Antagonistische, wie es Carl Schmitt, Chantal Mouffe und (allerdings auf andere Weise) Ernesto Laclau sowie Jacques Rancière denken? Oder das Kollektive, Ereignishafte, Emanzipatorische, wie es von Arendt konzipiert wird? Und inwiefern ist der politökonomische Ansatz der urban studies von einer solchen Konzeption des Politischen betroffen? Schließlich: Lässt sich mit einem postmarxistischen Ansatz eine Annäherung an das Wesen des Politischen finden? Wie lässt sich die Frage nach dem Politischen mit Marx verbinden? Mit unserem Themenheft möchten wir nicht nur dazu beitragen, solche Fragen in der Stadtforschung (weiter) zu diskutieren, sondern auch, dass mit ihnen neu verhandelt wird, was kritische Stadtforschung ist, was sie sein kann und sein will und welche gesellschaftstheoretischen Konzepte in diesem Rahmen diskutiert werden und Bezüge liefern.

\section{Endnoten}

[1] Als Überblickswerke im deutschen Sprachraum lassen sich nennen: Bröckling/ Feustel 2010, Bedorf/Röttgers 2010 sowie Flügel/Heil/Hetzel 2004.

[2] Dass sie damit selbst ein postpolitisches Konzept vorschlägt - zumindest dann, wenn Oliver Marcharts Konzeption des Politischen als Antagonismus gefolgt wird (2010; 2013) -, ist in der Debatte über einen ,agonistischen Pluralismus', die seit einigen Jahren insbesondere in der englischsprachigen Planungstheorie geführt wird (vgl. Bond 2011, Bäcklund/Mäntysalo 2010, Ploger 2004), bisher weitgehend übersehen worden.

[3] Dies hat zu massiven Vorwürfen gegen Arendt geführt, sie habe die Ungleichen und die vom griechischen Stadtstaat Ausgeschlossenen vergessen (vgl. Benhabib 1996). 


\section{Literatur}

Allmendinger, Phil / Haughton, Graham (2012): Postpolitical spatial planning in England: a crisis of consensus? In: Transactions of the Institute of British Geographers 37/1, 89-103.

Arendt, Hannah (1960): Vita Activa oder vom tätigen Leben. Stuttgart: Kohlhammer Verlag.

Arendt, Hannah (1994 [1961]): Zwischen Vergangenheit und Zukunft. Übungen im politischen Denken I. München: Piper Verlag.

Badiou, Alain (2010 [1985]): Ist Politik denkbar? Moral provisoire \#1. Berlin: Merve Verlag.

Bäcklund, Pia / Mäntysalo, Raine (2010): Agonism and institutional ambiguity. In: Planning Theory 9/4, 333-350.

Bedorf, Thomas / Röttgers, Kurt (2010): Das Politische und die Politik. Berlin: Suhrkamp Verlag.

Benhabib, Seyla (1996): The Reluctant Modernism of Hannah Arendt. Thousands Oaks: Sage.

Bond, Sophie (2011): Negotiating a 'democratic ethos.' Moving beyond the agonistic communicative divide. In: Planning Theory 10/2, 161-186.

Brenner, Neil / Theodore, Nik (Hg.) (2002): Spaces of Neoliberalism. Urban Restructuring in North America and Western Europe. Oxford: Blackwell.

Bröckling, Ulrich / Feustel, Robert (Hg.) (2010): Das Politische denken. Zeitgenössische Positionen. Bielefeld: transcipt Verlag.

Crouch, Colin (2008): Postdemokratie. Frankfurt a. M.: Suhrkamp Verlag.

Derrida, Jacques (2002): Politik der Freundschaft. Frankfurt a. M.: Suhrkamp Verlag.

Dikeç, Mustafa (2005): Space, politics, and the political. In: Environment and Planning D 23, 171-188.

Dikeç, Mustafa (2013): Beginners and equals: political subjectivity in Arendt and Rancière. In: Transactions of the Institute of British Geographers 38/1, 78-90.

Flügel, Oliver / Heil, Reinhard / Hetzel, Andreas (Hg.) (2004): Die Rückkehr des Politischen. Darmstadt: Wissenschaftliche Buchgesellschaft.

Foucault, Michel (2004a): Geschichte der Gouvernementalität I. Sicherheit, Territorium, Bevölkerung. Frankfurt a. M.: Suhrkamp Verlag.

Foucault, Michel (2004b): Geschichte der Gouvernementalität II. Die Geburt der Biopolitik. Frankfurt a. M.: Suhrkamp Verlag.

Füller, Henning / Michel, Boris (2012): Die Ordnung der Räume. Münster: Westfälisches Dampfboot.

Gauchet, Marcel / Lefort, Claude (1990): Über die Demokratie: Das Politische und die Instituierung des Gesellschaftlichen. In: Ulrich Rödel (Hg.), Autonome Gesellschaft und libertäre Demokratie. Frankfurt a. M.: Suhrkamp Verlag, 89-122.

Künkel, Jenny / Mayer, Margit (Hg.) (2012): Neoliberal Urbanism and Its Contestations: Crossing Theoretical Boundaries. Basingstoke: Palgrave Macmillan.

Laclau, Ernesto (1990): New Reflections on the Revolution of Our Time. London: Verso.

Laclau, Ernesto / Mouffe, Chantal (1991 [1985]): Hegemonie und radikale Demokratie. Zur Dekonstruktion des Marxismus. Wien: Passagen Verlag.

Lacoue-Labarthe, Philippe / Nancy, Jean-Luc (1997 [1990]): Retreating the Political. London/New York: Routledge.

Lefort, Claude (2007 [1999]): Complications. Communism and the Dilemmas of Democracy. New York: Columbia Press University.

Leitner, Helga / Peck, Jamie / Sheppard, Eric (Hg.) (2007): Contesting Neoliberalism. Urban Frontiers. New York: Guilford Press.

Marchart, Oliver (2010): Die Politische Differenz. Berlin: Suhrkamp Verlag.

Marchart, Oliver (2013): Das unmögliche Objekt. Berlin: Suhrkamp Verlag.

Mouffe, Chantal (1993): The Return of the Political. London: Verso.

Mouffe, Chantal (2000): Carl Schmitt and the Paradox of Liberal Democracy. In: dies. (Hg.), The Democratic Paradox. London: Verso, 36-59.

Mouffe, Chantal (2007 [2005]): Über das Politische. Frankfurt a. M.: Suhrkamp Verlag. Ploger, John (2004): Strife: urban planning and agonism. In: Planning Theory 3/1, 71-92. Purcell, Mark (2013): The Down-Deep Delight of Democracy. Malden: Wiley-Blackwell. Rancière, Jacques (2002 [1995]): Das Unvernehmen. Frankfurt a. M.: Suhrkamp Verlag. 
Roskamm, Nikolai (2012): Das Reden vom Raum. Zur Aktualität des ,Spatial Turn“ Programmatik, Determinismus und ,sozial konstruierter Raum‘. In: PERIPHERIE 126/127, 171-189.

Schmitt, Carl (1932): Der Begriff des Politischen. München/Leipzig: Duncker \& Humblot.

Schmitt, Carl (1933): Staat, Bewegung, Volk. Die Dreigliederung der politischen Einheit. Hamburg und Wandsbek: Hanseatische Verlagsanstalt.

Swyngedouw, Erik (2007): The post-political city. In: Guy Baeten (Hg.), Urban Politics Now. Re-imagining Democracy in the Neoliberal City. Rotterdam: NAi Publishers, 58-76.

Swyngedouw, Erik (2009): The antinomies of the postpolitical city: in search of a democratic politics of environmental production. In: International Journal of Urban and Regional Research 33/3, 601-620.

Žižek, Slavoj (2002): Revolution at the Gates. A Selection of Writings from February to October 1917. V.I. Lenin. London: Verso.

Žižek, Slavoj (2010 [1999]): Die Tücke des Subjekts. Frankfurt a. M.: Suhrkamp Verlag. 